\title{
Combining cow and bull reference populations to increase accuracy of genomic prediction and genome-wide association studies
}

\author{
M. P. L. Calus, ${ }^{1}$ Y. de Haas, and R. F. Veerkamp \\ Animal Breeding and Genomics Centre, Wageningen UR Livestock Research, 8200 AB Lelystad, the Netherlands
}

\begin{abstract}
Genomic selection holds the promise to be particularly beneficial for traits that are difficult or expensive to measure, such that access to phenotypes on large daughter groups of bulls is limited. Instead, cow reference populations can be generated, potentially supplemented with existing information from the same or (highly) correlated traits available on bull reference populations. The objective of this study, therefore, was to develop a model to perform genomic predictions and genome-wide association studies based on a combined cow and bull reference data set, with the accuracy of the phenotypes differing between the cow and bull genomic selection reference populations. The developed bivariate Bayesian stochastic search variable selection model allowed for an unbalanced design by imputing residuals in the residual updating scheme for all missing records. The performance of this model is demonstrated on a real data example, where the analyzed trait, being milk fat or protein yield, was either measured only on a cow or a bull reference population, or recorded on both. Our results were that the developed bivariate Bayesian stochastic search variable selection model was able to analyze 2 traits, even though animals had measurements on only 1 of 2 traits. The Bayesian stochastic search variable selection model yielded consistently higher accuracy for fat yield compared with a model without variable selection, both for the univariate and bivariate analyses, whereas the accuracy of both models was very similar for protein yield. The bivariate model identified several additional quantitative trait loci peaks compared with the single-trait models on either trait. In addition, the bivariate models showed a marginal increase in accuracy of genomic predictions for the cow traits (0.01-0.05), although a greater increase in accuracy is expected as the size of the bull population increases. Our results emphasize that the chosen value of priors in Bayesian genomic prediction models are especially important in small data sets.
\end{abstract}

Received July 31, 2012.

Accepted June 14, 2013.

${ }^{1}$ Corresponding author: mario.calus@wur.nl
Key words: genomic selection, genome-wide association study, multitrait, reference population

\section{INTRODUCTION}

Genotyping animals for genome-wide dense SNP markers is becoming cheaper every year, leading to the recent implementation of genomic selection in dairy cattle breeding programs in many countries (Hayes et al., 2009; Calus, 2010). Genomic selection is based on predicting breeding values from estimated SNP effects. The SNP effects are estimated in a reference population of animals with known genotypes and phenotypes (Meuwissen et al., 2001). In most implementation strategies in dairy cattle breeding programs, the phenotypes used are estimated breeding values of proven bulls, based on many daughter records. The heritability of the phenotypes used to estimate the direct genomic values (DGV) has an important effect on the reliability of the DGV (Daetwyler et al., 2008; Goddard, 2009). Therefore, using highly accurate phenotypes derived from EBV of proven bulls helps to maximize the reliability of DGV.

Over and above the recent focus on the application of genomic selection for routinely measured traits, where reliable breeding values based on daughter information are available for reasonable numbers of bulls, genomic selection holds the promise to be particularly beneficial for traits that are difficult or expensive to measure. This is particularly true for those traits where measuring (large) daughter groups on all bulls considered for selection is limited because of costs or difficulty of measurement. When the cost of measuring phenotypes is high compared with the genotyping cost per animal, an alternative is to generate a reference population with cow records. In that case, records from a limited number of cows are obtained, as well as genotypes, rather than using genotypes of their sires. Examples include analyses for susceptibility to paratuberculosis (Johne's disease; Kirkpatrick et al., 2011), energy balance (Verbyla et al., 2010), and progesterone profiles (Berry et al., 2012).

Traditionally, selection for difficult or expensive-tomeasure traits was performed using indicator traits. 
With the emerging possibility to phenotype and genotype a limited cow reference population for such traits, an interesting alternative approach is to simultaneously use the difficult-to-measure and indicator trait information together in multitrait genomic prediction (Calus and Veerkamp, 2011; Buch et al., 2012). Provided that a limited cow reference population for a specific trait is available, additional information on records for correlated traits can be used, as well as their genotypes. Correlated traits are likely to be measured on a large scale. One example of a pair of correlated traits is DMI that is measured only on a limited number of cows and BCS and conformation traits that are measured on a large scale throughout the population (Koenen and Veerkamp, 1998). In deterministic predictions, a multitrait approach for a situation where both cow direct measures and bull indicator trait phenotypes are used has been shown to have advantage over a single-trait approach in terms of the accuracy of genomic prediction (Calus et al., 2013). Thus far, there has been no demonstration of genomic prediction models that are able to estimate effects for 2 traits, where one trait is only measured on a limited cow reference population and the other correlated indicator trait is measured on daughters of genotyped bulls.

The objective of this study was to develop a Bayesian model to perform genomic predictions and genomewide association studies (GWAS) based on a combined cow and bull reference data set, with different traits being measured with different accuracies in the cow and the bull reference populations. The performance of this model is demonstrated using a real data example, where the analyzed trait was measured only on a cow or a bull reference population, or recorded on both. The traits considered were milk fat and protein yield. Those traits were chosen because of the availability of data, and to allow demonstration of the model based on wellknown traits. To allow comparison of the accuracy of genomic prediction obtained with the Bayesian model, accuracy of genomic prediction was also computed for a more commonly used model that uses genomic relationships between animals.

\section{MATERIALS AND METHODS}

\section{Phenotypes}

For this study, test-day records for milk, fat, and protein yields from 1,609 first-lactation Holstein heifers were used. Heifers originated from 4 countries: Ireland (Teagasc, Moorepark Dairy Production, Oak Park, Carlow), United Kingdom (Scottish Agricultural College, Edinburgh), the Netherlands (Wageningen University and Research Centre Livestock Research,
Wageningen), and Sweden (Swedish University of Agricultural Science, Uppsala). The phenotypes were preadjusted to account for mean lactation curve, herd, nutritional treatment, milking frequency, year-month of milk test by management group, and experimental treatments (for a full description, see Veerkamp et al., 2012). Average fat and protein yield of predicted values for wk 3 to 15 were used as phenotypes in our current study, for all animals with 10 or more observations in this period in the data set.

Daughter yield deviations (DYD) were available as well as associated reliabilities for 305-d fat and protein yield on 296 Holstein-Friesian dairy bulls evaluated by the Irish Cattle Breeding Federation (Bandon, Co. Cork, Ireland) in the July 2011 domestic genetic evaluations. Daughter yield deviations are estimated in Ireland using a repeatability animal model across the first 5 lactations (Berry et al., 2007). Effective daughter contributions (EDC; Fikse and Banos, 2001) were, on average, 2,043, ranging from 1.3 to 61,033 . Of those 296 bulls, 117 had daughters in the cow data set, varying from 1 to 40 .

\section{Genotypes}

The 1,609 heifers with phenotypes and the 296 bulls with DYD were all genotyped and were left in the data after editing the available genotype data. All those animals were genotyped using the Illumina BovineSNP50 BeadChip (Illumina Inc., San Diego, CA) containing 54,001 SNP. The pedigree was known for all genotyped animals. Quality control checks on the SNP included that the following criteria were met: (1) GC score $>0.20$ and GT score $>0.55,(2)$ call rate $>95 \%$, (3) minor allele frequency $>0.01$ in each country, and (4) no extreme deviation from Hardy-Weinberg Equilibrium (i.e., $\left.\chi^{2}<600\right)$. The GenCall (GC; Illumina Inc.) score and GenTrain (GT; Illumina Inc.) score are quality measures on the genotype calls from the genotyping assay. After the quality control edits 36,346 SNP on the 29 autosomes remained. Single nucleotide polymorphisms on the $\mathrm{X}$ chromosome were not included. Checks for Mendelian inconsistencies between pedigree and SNP data were performed for all genotyped parentoffspring pairs and among sibs (Calus et al., 2011), and animals with inconsistent pedigree and SNP information were removed. Missing genotypes were imputed using BEAGLE software (Browning and Browning, 2007). Chromosome number and locations of the SNP were obtained from the UMD3.1 bovine genome assembly (http://bovinegenome.org/cgi-bin/gbrowse/ bovine_UMD31/) from the University of Maryland (College Park). 


\section{Models}

Estimation of SNP Effects. The applied model to estimate SNP effects is referred to as Bayesian stochastic search variable selection (BSSVS; e.g., Verbyla et al., 2009). The general (multitrait) model was

$$
y_{i j}=\mu_{j}+\sum_{k=1}^{n l o c} \sum_{l=1}^{2} \mathrm{SNP}_{i j k l}+e_{i j},
$$

where $y_{i j}$ is the phenotypic record for trait $j$ (being the cow or the bull trait) of animal $i, \mu_{j}$ is the overall mean for trait $j$, nloc is the number of evaluated marker loci, $\mathrm{SNP}_{i j k l}$ is a random effect for allele $l$ on trait $j$ at locus $k$ of animal $i$, and $e_{i j}$ is a random residual for trait $j$ of animal $i$. The residual covariance between the analyzed cow and bull trait were always zero because little to no overlap existed between the cow data and the data used to estimate breeding values for the bulls.

The model is described in detail by Calus and Veerkamp (2011). Briefly, SNP effects (in the general model, denoted as $\left.\mathrm{SNP}_{i j k l}\right)$ are estimated as $q_{i j k l} \times v_{j k}$, following Meuwissen and Goddard (2004), where $q_{i j k l}$ is the size of the effect of allele $l$ at locus $k$ and $v_{j k}$ is a scaling factor in the direction vector for locus $k$ that scales the effect at locus $k$ for trait $j$. The variance of the direction vector $\mathbf{v}_{. k}$, is denoted as $\mathbf{V}$. The prior distribution for $\mathbf{V}[\mathrm{p}(\mathbf{V} .$.$) ; dots indicate the positions in$ the matrix corresponding to either trait] was as follows:

$$
\mathrm{p}(\mathbf{V} . .)=\chi^{-2}\left(\mathbf{S}_{0(. .)}, 10\right),
$$

where $\mathbf{S}_{0(n o)}$ (the scale matrix) is chosen such that it reflects the total genetic (co)variance between traits $n$ and $o$, divided by the total number of SNP; V.. was sampled from the following conditional $m$ variateinverted Wishart $\left(\mathrm{IW}_{m}\right)$ distribution with nloc +10 degrees of freedom:

$$
\text { V..|v.., I. } \sim \operatorname{IW}_{m}\left(\mathbf{S}_{\mathbf{0}(.)}+\mathbf{S Z}_{(. .)}, \text {nloc }-m-1+10\right) \text {, }
$$

where $\mathbf{S Z}_{(. .)}=\sum_{k=1}^{\text {nloc }} \mathbf{v}_{. k}^{\prime} \mathbf{v}_{. k}, \mathbf{S Z}_{(. .)}$is the sum of squares of the direction vectors $\mathbf{v}_{. k}$, and 10 is the number of degrees of freedom for the prior distribution, following the suggestion by Meuwissen and Goddard (2004).

Per locus, a single QTL indicator $\left(I_{k}\right)$ was sampled, to indicate per locus whether its effects were sampled from a distribution with large $\left(I_{k}=1\right)$ or small $\left(I_{k}=0\right)$ effects; $I_{k}$ was sampled from

$$
\begin{gathered}
I_{k} \mid \mathbf{v}_{. k}, \mathbf{V} \sim \operatorname{Bernoulli}\left(\phi\left(\mathbf{v}_{. k} ; \mathbf{0}, \mathbf{V}\right) \times \operatorname{Pr}\left(I_{k}=1\right) /\right. \\
\left\{\phi\left(\mathbf{v}_{. k} ; \mathbf{0}, \mathbf{V}\right) \times \operatorname{Pr}\left(I_{k}=1\right)+\phi\left(\mathbf{v}_{. k} ; \mathbf{0}, \mathbf{V} / 100\right)\right. \\
\left.\left.\times\left[1-\operatorname{Pr}\left(I_{k}=1\right)\right]\right\}\right),
\end{gathered}
$$

where $\operatorname{Pr}\left(I_{k}=1\right)$ is the prior probability that the effects at a locus belongs to the distribution with large effects. Here we used a value of 0.001 for $\operatorname{Pr}\left(I_{k}=1\right)$. Whenever $I_{k}=1\left(I_{k}=0\right)$, v.. was sampled using a variance equal to $\mathbf{V}(\mathbf{V} / 100)$. Posterior probabilities per locus are computed as the average of $I_{k}$ across all cycles after the burn-in.

The BSSVS model is implemented using residual updating. Residual updating implies that for each of the loci, the conditional phenotype of an animal is calculated as the sum of its current residual and the current estimate of its genotype at the evaluated locus (Legarra and Misztal, 2008). For missing records, however, no residuals are available from the data. As explained by Calus and Veerkamp (2011), our multitrait implementation imputes those missing residuals for animals with missing records, to obtain a balanced data design to estimate the SNP effects. In the application presented here, residuals are imputed for the first trait for all bulls and for the second trait for all cows. Imputed residuals for trait $j$ are simply drawn from $N\left(0, \hat{\sigma}_{e_{j}}^{2}\right)$, where $\hat{\sigma}_{e_{j}}^{2}$ is the current estimate of the residual variance for trait $j$. Residual variances for the second trait were weighted for each bull by the reciprocal of their individual EDC. Imputed residuals were used to construct $\mathrm{y}^{*}$, being phenotypic records adjusted for all other effects, to estimate the SNP effects using residual updating. Construction of $\mathrm{y}^{*}$ is completed for imputed records in the same way as for observed records, by adding up the last estimate for the genotype at the locus that is currently evaluated. Estimation of SNP effects with the (bivariate) BSSVS model is described in detail by Calus and Veerkamp (2011). All BSSVS models were performed using Gibbs sampling with residual updating. In total, 5 different chains with 50,000 cycles were run, discarding 10,000 for burn-in.

Genomic-Relatedness-Matrix REML. To allow comparison of results of the BSSVS model, genomic breeding values were also predicted using univariate and bivariate versions of a genomic BLUP-type model. This model is here termed genomic-relatedness-matrix REML (GREML), because it estimates genomic breeding values and variance components simultaneously using REML. The bivariate version of this model was as follows:

$$
y_{i j}=\mu+g_{i j}+e_{i j}
$$

where $\mu$ is an overall mean and $y_{i j}, g_{i j}$, and $e_{i j}$ are, respectively, the phenotype, direct genomic value, and residual for trait $j$ of animal $i$. Additive genetic and residual effects were assumed to be distributed as $\mathbf{g}$ $\sim N(\mathbf{0}, \mathbf{G R M} \otimes \mathbf{G})$ and $\mathbf{e} \sim N(\mathbf{0}, \mathbf{I} \otimes \mathbf{R})$, where $\mathbf{g}$ is a matrix of direct genomic values, $\mathbf{e}$ is a matrix of 
residuals, GRM is the genomic relationship matrix, $\mathbf{G}$ is a matrix with additive genetic (co)variances, $\mathbf{I}$ is an identity matrix, and $\mathbf{R}$ is a diagonal matrix with residual variances. The genomic relationship matrix was calculated following VanRaden (2008), applying changes to the elements to account for sampling variance as proposed by Yang et al. (2010). Full details of this procedure are described in Pszczola et al. (2012b).

Estimation of Genetic Correlations Between Traits. To facilitate assessment of expected benefit from adding the bull information into the analyses, we estimated genetic correlations between bulls and cows, separately for fat and protein yield. The following bivariate model was used to estimate both correlations:

$$
y_{i j}=\mu+a_{i j}+e_{i j},
$$

where $\mu$ is an overall mean and $y_{i j}, a_{i j}$, and $e_{i j}$ are, respectively, the phenotype, additive genetic effect, and residual for trait $j$ of animal $i$. Additive genetic and residual effects were assumed to be distributed as $\mathbf{a} \sim$ $N(\mathbf{0}, \mathbf{A} \otimes \mathbf{G})$ and $\mathbf{e} \sim N(\mathbf{0}, \mathbf{I} \otimes \mathbf{R})$, where $\mathbf{a}$ is a matrix of additive genetic effects, $\mathbf{e}$ is a matrix of residuals, $\mathbf{A}$ is the numerator relationship matrix calculated from the pedigree information, $\mathbf{G}$ is a matrix with additive genetic (co)variances, $\mathbf{I}$ is an identity matrix, and $\mathbf{R}$ is a diagonal matrix with residual variances. All described (co)variances were estimated simultaneously with the additive genetic effects, but the residual covariance between the cow and bull traits was zero. Residual variances used for individual bulls were weighted using their EDC, similarly as described above for the BSSVS model. This model was fitted using Gibbs sampling. For both fat and protein, 1 chain of 1,000,000 cycles was run, discarding 200,000 for burn-in.

\section{Weights for Different Information Sources}

Weights were used for individual phenotypic records in all models. All preadjusted cow records received an equal weight of 1 (i.e., we assumed that the cow records were each based on similar amounts of information). Daughter-yield deviations were used as phenotypes for the bulls. The weights used for individual DYD were EDC. Those EDC were calculated from the reliability (REL) of the equivalent breeding values as $\alpha \mathrm{REL} /(1$ - REL), where $\alpha=\left(4-h^{2}\right) / h^{2}$ (Fikse and Banos, 2001). The EDC were used as weights for the DYD in all analyses, by dividing the residual variance for each bull by its EDC value.

\section{Scenarios and Assessment of Model Performance}

Different scenarios were considered to investigate the added value of including bull information with the cow reference population, and to evaluate the performance of the presented model. The first scenario included only cow records in the reference population. The second scenario included only bull records in the reference population. The third scenario included both cow and bull records in the reference population.

To assess the performance of the model for GWAS, for each scenario 5 different Gibbs chains were run. Posterior QTL probabilities of each chain were used to calculate Bayes factors (BF) for each SNP as follows:

$$
\mathrm{BF}=\frac{\operatorname{Pr}\left(H_{1} \mid y\right)}{1-\operatorname{Pr}\left(H_{1} \mid y\right)} \div \frac{\operatorname{Pr}\left(H_{1}\right)}{1-\operatorname{Pr}\left(H_{1}\right)},
$$

where $H_{1}$ is the hypothesis that the marker is linked to a QTL, $\operatorname{Pr}\left(H_{1} \mid y\right)$ is the posterior probability of the hypothesis, $y$ represents the data, and $\operatorname{Pr}\left(H_{1}\right)$ is the prior probability of the hypothesis; $1-\operatorname{Pr}\left(H_{1} \mid y\right)$ and 1 $\operatorname{Pr}\left(H_{1}\right)$ represent the posterior and prior probability for the alternative hypothesis, respectively. Because many SNP are closely linked and different sets of SNP might give equivalent good fit to the data in different chains, the average Bayes factor of a window of 9 SNP (a SNP plus its 4 closest neighboring SNP on either side) was also used. These average BF are used to smooth the results (i.e., by averaging $\mathrm{BF}$, evidence for presence of a QTL within a window is accumulated, which might reveal regions with QTL that are missed otherwise). We used 10 as a threshold value for regions that had a very clear association with the analyzed trait. Bayes factors $>10$ indicate "strong evidence against the null hypothesis" (Jeffreys, 1961), in our case indicating strong evidence that this particular locus has an association with the analyzed trait.

Performance in terms of genomic prediction was assessed using the same 3 scenarios. To facilitate calculation of prediction accuracy with cross-validation, the cow data was split in 10 subsets based on country and experimental treatment within country (Table 1). Large subgroups in Ireland and the Netherlands were split in half at random, such that all groups contained at most 200 animals (Table 1). For the scenario using only a cow trait and for the scenario using a cow and a bull trait, 10 analyses were performed, where each of the cow subsets was omitted once. The analyses including both a cow and a bull trait [i.e., when both traits were used to estimate the SNP effects (BSSVS) or directly the genomic breeding values (GREML)] always used the records of all bulls. The analyses including only a bull trait were run just once using all bulls. For all scenarios, prediction accuracy was calculated for each of the 10 cow subset as the Pearson correlation between observed phenotypes $(y)$ and predicted DGV, divided 
Table 1. Cross-validation groups defined within country

\begin{tabular}{llc}
\hline Group & Country (herd) & $\begin{array}{c}\text { Number } \\
\text { of animals }\end{array}$ \\
\hline 1 & Scotland (Langhill) & 98 \\
2 & & 108 \\
3 & & 99 \\
4 & the Netherlands ('t Gen) ${ }^{2}$ & 108 \\
5 & & 197 \\
6 & & 197 \\
7 & Sweden (SLU $^{3}$ ) & 135 \\
8 & Ireland (Teagasc) & 181 \\
9 & & 189 \\
10 & & 189 \\
\hline
\end{tabular}

${ }^{1}$ Groups 1 to 4 were defined as treatment groups in the diet by genetic line $(2 \times 2)$ experimental setup (for details, see Banos et al., 2012) The herd was from Teagasc, Moorepark Dairy Production (Oak Park, Carlow, Ireland)

${ }^{2}$ Group 7 contained bull dams. Groups 5 and 6 were created by randomly splitting the remaining 394 animals. The herd was from Wageningen University and Research Centre Livestock Research (Wageningen, the Netherlands).

${ }^{3} \mathrm{SLU}=$ Swedish University of Agricultural Sciences (Uppsala, Sweden). ${ }^{4}$ Groups 9 and 10 were created by randomly splitting the 378 animals from Teagasc (Moorepark Dairy Production, Oak Park, Carlow, Ireland).

by the square root of the heritability. This correlation (r) can be represented as follows:

$$
\mathrm{r}=\frac{\operatorname{cov}(y, \widehat{\mathrm{DGV}})}{\sqrt{\operatorname{var}(y) \operatorname{var}(\widehat{\mathrm{DGV}})}}
$$

To adjust this correlation to obtain an accuracy of the DGV, $\operatorname{var}(y)$ needs to be replaced by the variance of the true breeding value $[\operatorname{var}(\mathrm{TBV})]$. Note that the expectation is that $\operatorname{cov}(y, \widehat{\mathrm{DGV}})=\operatorname{cov}(\mathrm{TBV}, \widehat{\mathrm{DGV}})$. The accuracy of the DGV is then as follows:

$$
\mathrm{r}_{\mathrm{DGV}}=\frac{\operatorname{cov}(\mathrm{TBV}, \widehat{\mathrm{DGV}})}{\sqrt{\operatorname{var}(\mathrm{TBV}) \operatorname{var}(\widehat{\mathrm{DGV}})}}=\frac{\operatorname{cov}(y, \widehat{\mathrm{DGV}})}{\sqrt{\operatorname{var}(y) \operatorname{var}(\widehat{\mathrm{DGV}})}} \times \frac{1}{h},
$$

where $h$ is the square root of the heritability, which is considered to be equal to $\sqrt{\operatorname{var}(\mathrm{TBV})} / \sqrt{\operatorname{var}(y)}$.

Next to the accuracy, also the coefficient of the regression of the phenotypes on the DGV was calculated, to assess bias in the estimates. This regression coefficient (rc) can be represented as follows:

$$
\mathrm{rc}=\frac{\operatorname{cov}(y, \widehat{\mathrm{DGV}})}{\operatorname{var}(\widehat{\mathrm{DGV}})} .
$$

Because the expectation is that $\operatorname{cov}(y, \widehat{\mathrm{DGV}})=\operatorname{cov}(\mathrm{TBV}, \widehat{\mathrm{DGV}})$, the expected value for $\mathrm{rc}$ is 1.0 when $\widehat{\mathrm{DGV}}$ are unbiased.

\section{RESULTS}

\section{Genetic Parameters}

Heritabilities for the cow traits, estimated with the pedigree based model, were 0.364 for fat and 0.347 for protein (Table 2). Estimated heritabilities for the bull traits were, as expected, close to 1.0, because the observations were generally highly reliable DYD based on many daughters. The genetic correlation between cows and bulls for fat and protein yield were moderate (0.586 and 0.645 , respectively). Both genetic correlations were significantly different from 1.0 (i.e., the $95 \%$ posterior confidence intervals were 0.35 to 0.90 and 0.36 to 0.91 for fat and protein, respectively).

\section{GWAS}

Manhattan plots of average BF for windows of 9 SNP are shown for fat (Figure 1) and protein (Figure 2) yield. Figures 1 and 2 reveal several clear putative QTL along the genome, and the number of putative QTL was larger, both for fat and protein, for the analyses that combined the cow and bull data. Per trait and analysis combination, the number of regions $(<3 \mathrm{Mb}$ in size) with an average $\mathrm{BF}>10$ were counted (Table 3 ). Those results show that the number of detected regions in the combined analysis was much greater than in the analyses including only the cow or bull information. In addition, poor (fat) or no overlap (protein) existed between the detected regions using either cow or bull information. The combined analyses, however, showed a much stronger overlap with the analyses using only the cow or bull information. This was expected, as no overlap existed between the cow and bull data, and

Table 2. Estimated heritabilities of fat and protein yield measured on cows and based on bull daughter yield deviations (DYD) ${ }^{1}$ and estimated genetic correlations (and their SD) between cows and bulls for fat and protein ${ }^{2}$

\begin{tabular}{lcc}
\hline Item & Fat & Protein \\
\hline Heritabilities & & \\
Cows & $0.364(0.051)$ & $0.347(0.051)$ \\
Bulls & $1.000(0.000)$ & $0.986(0.006)$ \\
Genetic correlations & $0.586(0.132)$ & $0.645(0.139)$ \\
\hline
\end{tabular}

${ }^{1}$ Heritabilities for bulls were calculated at the level of the average effective daughter contribution $(2,043)$.

${ }^{2}$ Estimates were obtained with 2 bivariate pedigree-based models using Gibbs sampling. 
Fat yield
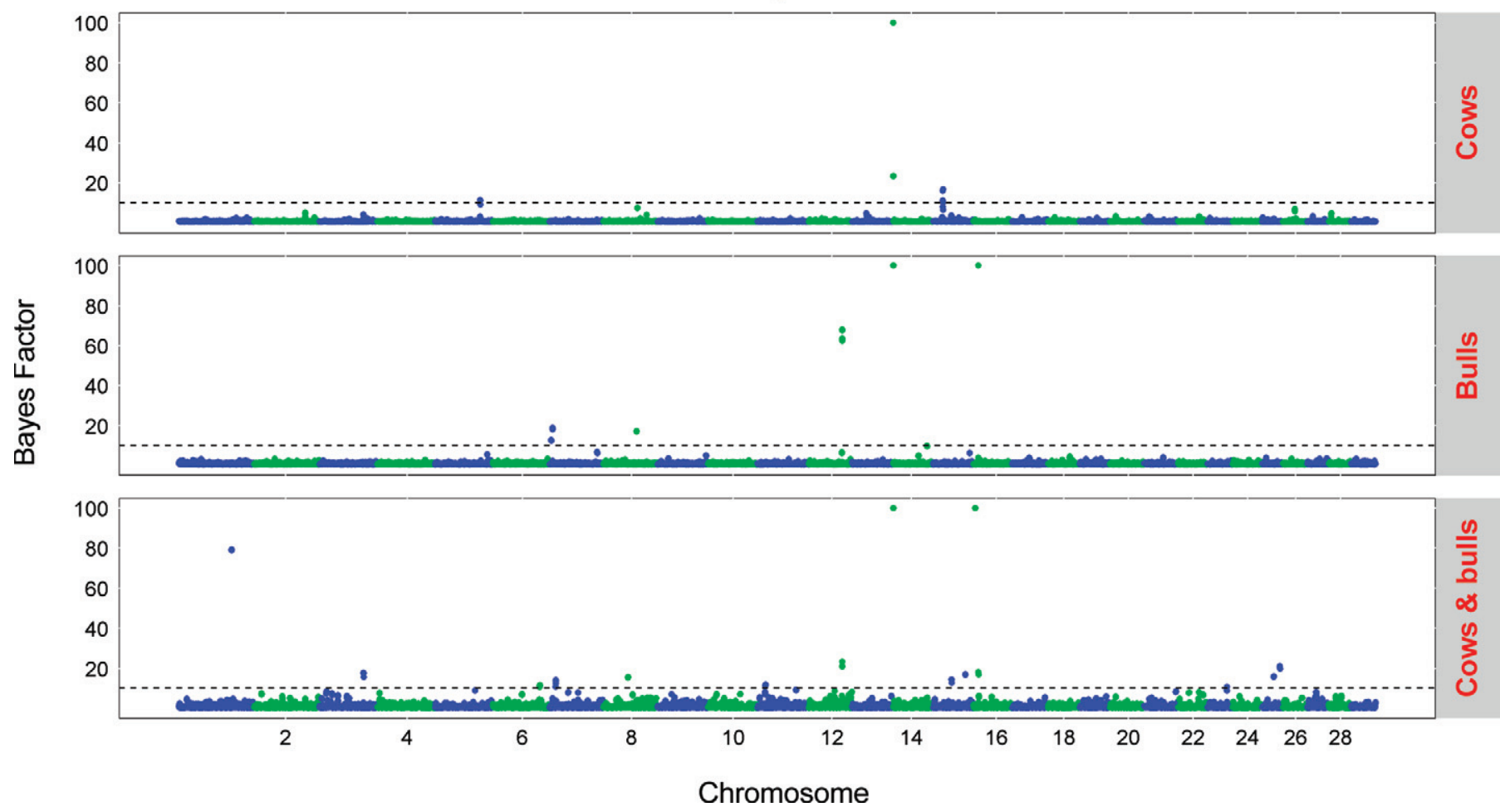

Figure 1. Bayes factors across the genome for fat yield, using cow, bull, or cow and bull data (Bayes factors >100 were set to 100). Color version available in the online PDF.

both the cow and the bull data sets obviously do overlap with the combined data.

\section{Genomic Prediction}

Genomic predictions were performed using 10 crossvalidation subsets of animals within the cow data. Results show that accuracies of prediction for both fat and protein were higher when the cow data were used, compared with when the bull data were used (Table 4). The model BSSVS yielded 0.07 to 0.11 higher accuracies for fat yield compared with GREML across the different data sets used. Accuracies for BSSVS and
GREML were very similar for protein. Simultaneously analyzing the bull and cow data in a bivariate analysis, compared with using the cow data alone, yielded increases in accuracy of 0.02 to 0.05 and 0.01 to 0.02 for fat and protein, respectively (Table 4).

Coefficients of regressions of cow phenotypes on their predicted DGV in all cases deviated substantially from 1.0 (Table 5). The model BSSVS yielded higher regression coefficients compared with GREML in all scenarios. Using only bull data in the reference population decreased the regression coefficients compared with the scenario where the reference population consisted of only cow data. Using the cow and bull data

Table 3. Numbers of regions $(<3 \mathrm{Mb}$ in size) in which effects with Bayes factors $>10$ were identified for fat and protein, using either the cow, bull, or combined data (diagonals), and the number of regions that were identified by 2 analyses (off-diagonals)

\begin{tabular}{llccc}
\hline \multirow{2}{*}{ Trait } & Data used & Cows & Bulls & $\begin{array}{c}\text { Cows } \\
\text { and bulls }\end{array}$ \\
\hline Fat & Cows & 3 & & \\
& Bulls & 1 & 5 & 15 \\
\multirow{2}{*}{ Protein } & Cows and bulls & 1 & 4 & \\
& Cows & 2 & & 12 \\
& Bulls & 0 & 2 & 12 \\
\hline
\end{tabular}


Table 4. Average accuracies (Acc.) and their SE of genomic predictions for fat and protein yields, obtained using Bayesian stochastic search variable selection (BSSVS) or genomic-relatedness-matrix REML (GREML), for various reference populations, calculated as the correlations between the estimated direct genomic values (DGV) and the phenotypes divided by the square root of the heritability, as estimated with the 2 bivariate pedigree-based models ${ }^{1}$

\begin{tabular}{|c|c|c|c|c|c|c|c|c|}
\hline \multirow{3}{*}{$\begin{array}{l}\text { Reference } \\
\text { population }\end{array}$} & \multicolumn{4}{|c|}{ Fat } & \multicolumn{4}{|c|}{ Protein } \\
\hline & \multicolumn{2}{|c|}{ BSSVS } & \multicolumn{2}{|c|}{ GREML } & \multicolumn{2}{|c|}{ BSSVS } & \multicolumn{2}{|c|}{ GREML } \\
\hline & Acc. & $\mathrm{SE}$ & Acc. & $\mathrm{SE}$ & Acc. & $\mathrm{SE}$ & Acc. & $\mathrm{SE}$ \\
\hline Cows & 0.333 & 0.070 & 0.226 & 0.064 & 0.193 & 0.052 & 0.198 & 0.052 \\
\hline Bulls & 0.244 & 0.054 & 0.173 & 0.053 & 0.152 & 0.052 & 0.156 & 0.053 \\
\hline Bulls and cows & 0.355 & 0.065 & 0.273 & 0.065 & 0.207 & 0.056 & 0.221 & 0.054 \\
\hline
\end{tabular}

${ }^{1}$ For each trait (i.e., fat or protein), predictions were based on only cow or bull data, or both combined.

simultaneously in the reference population improved the regression coefficients for both traits.

\section{DISCUSSION}

The objective of this study was to develop a model to perform GWAS and genomic predictions based on a combined cow and bull reference data set, with different but highly correlated traits being measured on the cows than on the daughters of the bulls. In this study, we mimicked this by analyzing fat (or protein) yield, where the same trait measured based on own performance of cows versus daughter yield deviation of bulls had a genetic correlation that was significantly lower than 1.0. Analyses on a data set with records of 1,609 cows and 296 bulls indicate that adding the bull information next to the cow information helped to improve the power for GWAS. Despite the increased number of QTL peaks identified, genomic prediction accuracies increased only marginally, most probably because the genetic correlation between the cow and bull traits was far from unity, and because the number

Protein yield

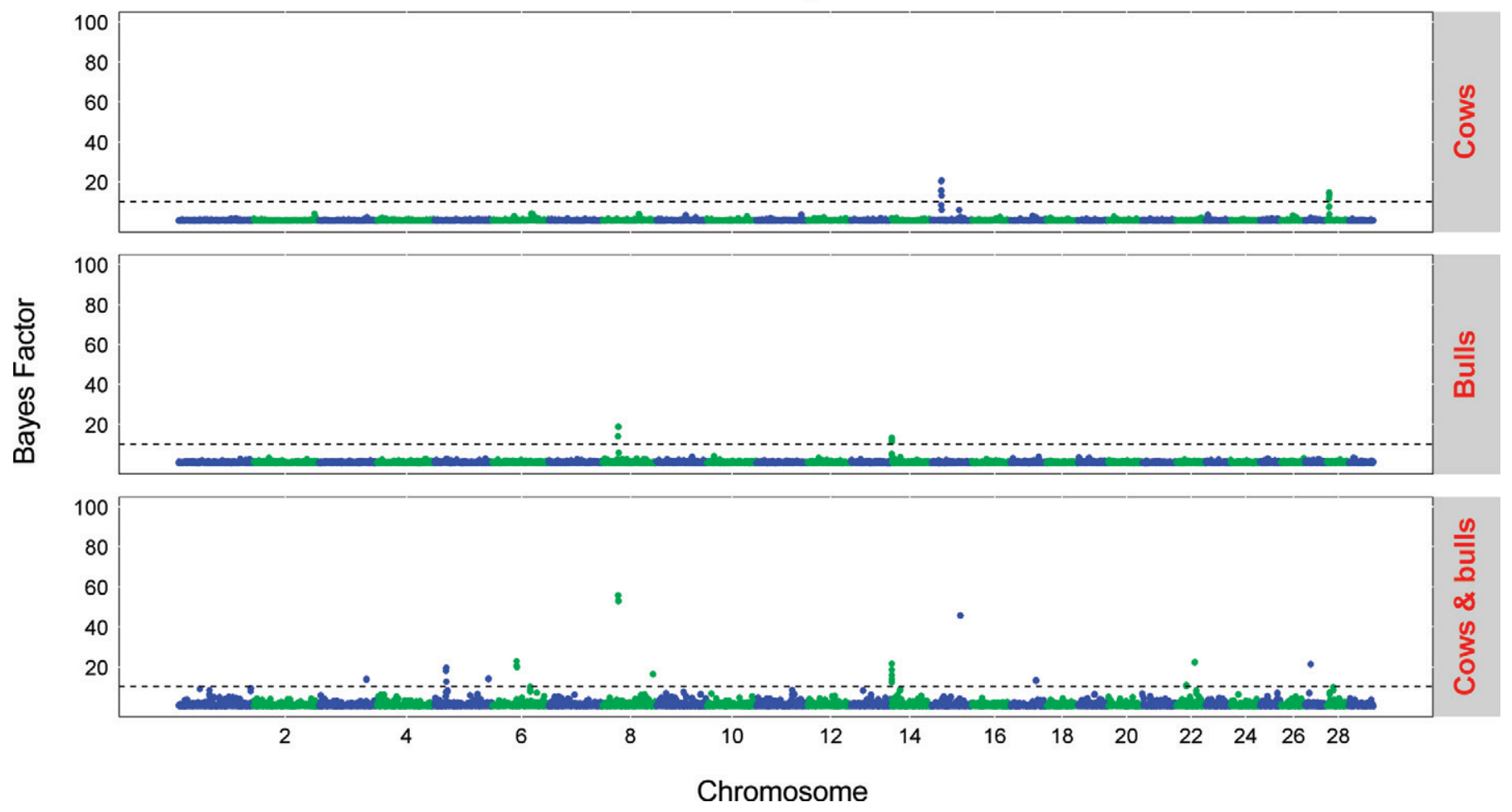

Figure 2. Bayes factors across the genome for protein yield, using cow, bull, or cow and bull data (Bayes factors $>100$ were set to 100 ). Color version available in the online PDF. 
Table 5. Slopes of the regressions (Regr.) and their SE of the phenotypes on genomic prediction for fat and protein yield, obtained using Bayesian stochastic search variable selection (BSSVS) or genomic-relatednessmatrix REML (GREML), for various reference populations ${ }^{1}$

\begin{tabular}{|c|c|c|c|c|c|c|c|c|}
\hline \multirow{3}{*}{$\begin{array}{l}\text { Reference } \\
\text { population }\end{array}$} & \multicolumn{4}{|c|}{ Fat } & \multicolumn{4}{|c|}{ Protein } \\
\hline & \multicolumn{2}{|c|}{ BSSVS } & \multicolumn{2}{|c|}{ GREML } & \multicolumn{2}{|c|}{ BSSVS } & \multicolumn{2}{|c|}{ GREML } \\
\hline & Regr. & $\mathrm{SE}$ & Regr. & $\mathrm{SE}$ & Regr. & SE & Regr. & $\mathrm{SE}$ \\
\hline Cows & 0.628 & 0.132 & 0.495 & 0.132 & 0.482 & 0.124 & 0.464 & 0.117 \\
\hline Bulls & 0.427 & 0.104 & 0.264 & 0.117 & 0.356 & 0.127 & 0.354 & 0.136 \\
\hline Bulls and cows & 0.676 & 0.130 & 0.583 & 0.139 & 0.547 & 0.165 & 0.529 & 0.133 \\
\hline
\end{tabular}

${ }^{1}$ For each trait (i.e., fat or protein), predictions were based on only cow or bull data, or both combined.

of added bull records was limited. To demonstrate the real potential of the BSSVS model combining cow and bull information, a much larger bull reference data set is required. Nevertheless, comparison of the results for fat yield obtained with the BSSVS and GREML models revealed that the BSSVS model not only gave more accurate predictions than GREML when only the cow or bull data was analyzed, but that this increase in accuracy was also obtained when both the cow and bull traits were included.

\section{Combining Cow and Bull Data}

For the traits that we considered, one would expect to observe near unity genetic correlations between the same trait measured in bulls and cows. However, the genetic correlations between cows and bulls were only 0.59 and 0.65 for fat and protein, respectively. These moderate genetic correlations mainly result from the fact that the cow traits only capture yields in the first 15 wk of the lactation, because for most cows detailed yield records were only available until 15 wk after calving, whereas the bull DYD reflect complete lactations.

In the models presented here, it is important to avoid double counting of information of cows that are included with their phenotypes in the analyses, and at the same time contribute to the DYD of the bulls that are included in the analysis. In our data, this double counting may arise through daughters of the 296 bulls that are included themselves, because they are producing in one of the included Irish herds while they have contributed to the Irish DYD of their sires, which are included in the analysis as well. In the data used, 60 of the 296 bulls included in the analyses in fact had, on average, 4.9 (with a maximum of 30 ) daughters in Ireland with phenotypes in the data. To get more insight in the relative contribution of those daughters to the bull DYD, the number of daughters for those bulls with data in the analyses was expressed as a percentage of their EDC values. This percentage was, on average, $0.4 \%$ with a maximum of $3.4 \%$. Because the DYD of those 60 bulls, on average, had reliabilities of $98.2 \%$, we expect that excluding their daughters with phenotypes used in our analyses, would not change their DYD and, therefore, also not affect the results. Nevertheless, in similar analyses where a significant proportion of the daughters of bulls are both included with their own phenotypes and do contribute to the DYD of their sires, double counting of the cow information may lead to biased results. To avoid this, either daughters of included bulls should be removed from the data or DYD should be estimated excluding information of daughters that are included as cows in the analysis. The latter approach is expected to be more powerful, as it still uses the genotypic information of the daughters.

\section{The Model}

The model that was developed and applied in our study samples a QTL indicator for each SNP locus to determine whether this locus has a big or small effect on the trait. This feature of the BSSVS model resulted in consistently higher accuracies for fat yield compared with GREML, due to the effects of the DGAT1 gene, as evidenced by posterior QTL probabilities being (close to) 1.0 in the DGAT1 region (results not shown). In the bivariate model, this QTL indicator is sampled for both traits simultaneously (Meuwissen and Goddard, 2004; Calus and Veerkamp, 2011). This implies that the model may detect a QTL that affects both traits or only one of the traits, because the model does not discriminate between QTL that affect only one or both traits. This limitation is not expected to greatly affect the results presented here, as the cow and bull traits studied here had a genetic correlation $>0.5$. As a result, the analysis on the combined cow and bull data revealed several additional QTL regions. Some of the additional regions clearly coincide with QTL regions that were reported in other studies. This is, for instance, the case for the QTL peak on chromosome 6 at $98.3 \mathrm{Mb}$ for fat yield (Wiener et al., 2000; Harder et al., 2006) and the peak on chromosome 6 at $51.9 \mathrm{Mb}$ for protein yield (Wiener 
Table 6. Additional QTL detected using the combined sire and cow analysis, and studies that report QTL coinciding with those additional QTL

\begin{tabular}{lrrrl}
\hline Trait & BTA & Start $^{1}$ & \multicolumn{1}{c}{ End $^{1}$} & QTL \\
Fat & 1 & 110.1 & 110.8 & \\
& 3 & 92.2 & 92.6 & \\
& 6 & 98.1 & 98.6 & Wiener et al. (2000); Harder et al. (2006) \\
& 7 & 12.4 & 13.1 & Ogorevc et al. (2009) \\
& 11 & 16.3 & 16.9 & \\
& 15 & 39.1 & 39.7 & \\
& 15 & 68.0 & 68.7 & \\
& 16 & 3.6 & 4.0 & \\
Protein & 23 & 40.7 & 40.7 & Ogorevc et al. (2009) \\
& 25 & 24.0 & 24.6 & \\
& 25 & 36.6 & 37.1 & \\
& 3 & 99.3 & 99.7 & \\
& 5 & 24.6 & 25.5 & \\
& 5 & 113.4 & 114.0 & Rodriguez-Zas et al. (2002) \\
& 6 & 51.7 & 52.1 & Wiener et al. (2000); Ron et al. (2001) \\
& 8 & 105.7 & 106.0 & \\
& 17 & 53.3 & 53.7 & Harder et al. (2006); Ogorevc et al. (2009) \\
& 22 & 21.0 & 21.3 & Ashwell et al. (2004); Ogorevc et al. (2009) \\
& 22 & 38.3 & 38.8 & \\
\hline
\end{tabular}

${ }^{1}$ Start and end indicate first and last position with Bayes factors $>10$ within regions $(<3 \mathrm{Mb}$ in size). Positions were obtained from the UMD3.1 bovine genome assembly (http://bovinegenome.org/cgi-bin/gbrowse/bovine_ UMD31/) from the University of Maryland (College Park).

${ }^{2}$ The QTL intervals reported in those studies overlapped with the intervals detected in our study.

et al., 2000; Ron et al., 2001). Out of the 11 additional identified QTL for fat, 3 coincided with QTL previously reported in the literature, whereas out of the 9 additional identified QTL for protein, 5 coincided with QTL previously reported in the literature (as summarized in Table 6). These results indicate that, although some of the additional QTL may be false positives, several of the additional QTL appear to be true because they can be validated based on existing literature.

An alternative strategy to sampling a QTL indicator for both traits simultaneously could be to sample a QTL indicator for each trait separately. An important unanswered question is which strategy is optimal. The main benefit of using a single QTL indicator is that evidence for the presence of a QTL for one trait may be increased by information from a correlated trait, as the prior variances depend on the genetic correlation. At the same time, this mechanism may force the genetic correlation on the estimates, even when the correlation at a specific locus is, in fact, opposite to the genetic correlation. This is illustrated with the estimated allele substitution effects for protein, using both the cow and bull data, which showed a strong correlation among each other, in line with the estimated genetic correlation (Figure 3). Using a similar model and pairs of milk yield traits within the cow data used in our study, van Binsbergen et al. (2012) found that across the whole genome, only a few SNP showed effects in the opposite direction compared with the genetic correlation. It should, however, be noted that the impact of the priors on the estimated effects is expected to decrease as sample size increases (Sorensen and Gianola, 2002; de los Campos et al., 2013), which indicates that this issue may at least partly be overcome when the data set used has substantially larger dimensions. Nevertheless, the impact of the prior variances and, in particular, the genetic correlation, on the obtained estimates in a limited data set such as the one used in the present study, is expected to be large. This also implies that incorrect prior variances may, in fact, be harmful for the predictions when the data are not powerful enough to override the prior. To test this, the cross-validations for the combined cow and sire data were repeated for both fat and protein, using a zero genetic correlation between the cow and bull trait in the prior variances. This resulted in accuracies (with standard errors) of $0.189(0.043)$ for fat and $0.160(0.054)$ for protein. This accuracy for fat was significantly lower than the corresponding accuracy obtained with the BSSVS model using the estimated genetic correlation to specify the prior covariances (Table 4). The accuracy for protein was also lower than the corresponding accuracy obtained with the BSSVS model using correct priors, but the difference was not significant. For both fat and protein, the accuracies were lower than those obtained with GREML, except for the scenario where only bull data was used for protein. These additional results stress that the chosen value of priors are especially important in small data sets (Sorensen and Gianola, 2002; de los Campos et al., 2013). 
Allele substitution effects

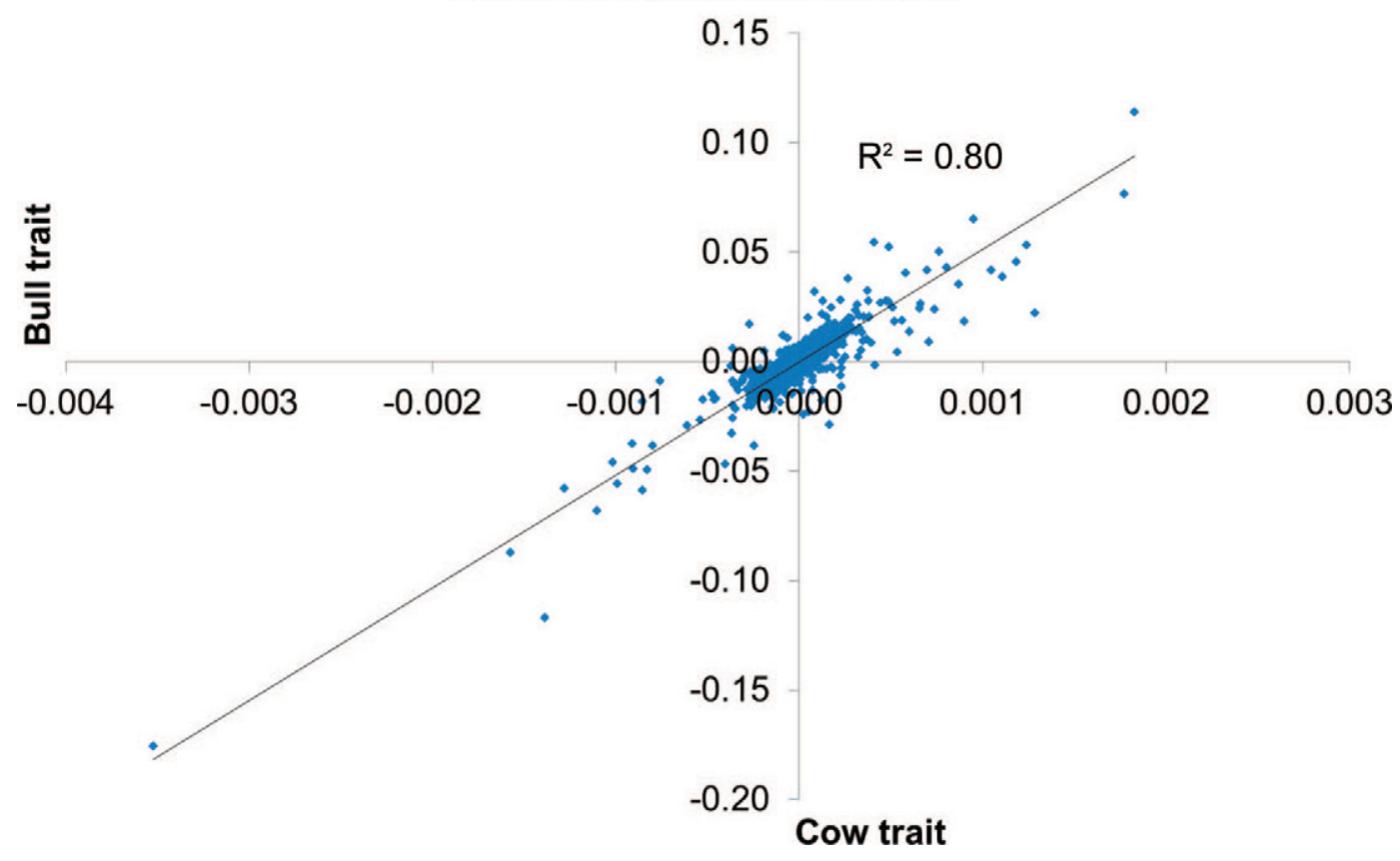

Figure 3. Allele substitution effects for protein yield, recorded as daughter yield deviations (DYD; bull trait) or cow phenotypes (cow trait), estimated with the combined cow and bull genome-wide association study (GWAS) analysis (i.e., including all animals), for 1 of 5 Gibbs chains. Color version available in the online PDF.

The genetic correlations between the cow and bull traits analyzed were 0.59 and 0.65 for fat and protein, respectively. For such genetic correlations, and for lower values of the genetic correlation, it is expected that a bivariate model yields more accurate predictions than a model that just treats all observations as if they belong to the same trait. Moreover, defining weights for the cow records that put them on the same scale as the bull records may not be trivial. Despite the fact that weights for different types of phenotypes have been formally derived (Garrick et al., 2009), those weights do depend on genetic parameters, whose estimates may be biased. The bivariate model circumvents any of these issues by explicitly modeling different scales per trait. In a study where genomic BLUP was used in different scenarios that combined DMI data across environments, it was also concluded that a multitrait model was preferred over a single-trait model when the pairwise genetic correlations between environments ranged from 0.36 to 0.74 (de Haas et al., 2012).

Several studies thus far have shown that multitrait genomic-prediction models yield higher accuracies than their single-trait counterparts (VanRaden and Sullivan, 2010; Calus and Veerkamp, 2011; Tsuruta et al., 2011). In all those cases, the increase in accuracy resulted from the fact that animals in the reference population had phenotypes for several traits. In the model that we presented here, animals had phenotypes for only 1 of the 2 traits. In the past, developing such models that used pedigree information was often challenging (e.g., Schaeffer et al., 1978; Ducrocq and Besbes, 1993), especially when weak genetic ties existed between the animals that had phenotypes for one versus the other trait (e.g., Mark et al., 2005). This problem may be alleviated in a genomic prediction model, because by using the genomic information, the connectedness between both groups of animals is improved compared with a pedigree-based model (Pszczola et al., 2012b). In a genomic prediction model, pairs of animals that have zero relationships in a relationship matrix calculated from pedigree may have nonzero genomic relationships. Although this group of genomic relationships mainly contains small values, many of these may exist, which helps to improve connectedness between groups of animals.

\section{Design of the Reference Population}

Coefficients of regression of cow phenotypes on their predicted DGV were in all situations substantially lower than the expected value of 1.0. The phenotypes used were obtained from 4 different countries, and preadjusted and standardized across environments and levels of different fixed effects. Despite these adjust- 
ments, apparently the data was still heterogeneous, thereby resulting in regression coefficients that deviate from unity. Such heterogeneity may be overcome by modeling the same traits recorded in different environments as different traits, which accommodates for the differences in scale across environments. This is also indirectly shown by our results. When only the bull trait was used, the regression coefficients deviated most from 1.0. When the bull trait was included next to the cow trait, the regression coefficient in fact became closer to 1.0 compared with the situation where only the cow trait was included.

Although the model was developed to complement analyses using data from limited cow reference populations, its potential application may be much wider than such scenarios alone. For traits routinely measured in dairy cattle populations, bull reference populations have been composed for several dairy cattle breeds. At some stage, all bulls from the historic population with highly accurate daughter-based breeding values and DNA material available for genotyping will have been added to the bull reference population. This limited availability of bulls with accurate breeding value may especially pose a limit to the potential accuracy of genomic selection for small breeds or populations, but eventually this may also be the case for mainstream breeds. One possibility to further increase the size of the reference population is collaboration across countries and organizations (Wiggans et al., 2008; Cromie et al., 2010; Lund et al., 2011). Thereafter, every year, a limited number of bulls that were initially selected based on their DGV and eventually reach an appropriate number of daughters in their index after several years of servicing, can be added. One suggested possibility to further increase the size and, thus, the power of reference populations is to add genotyped cows ( $\mathrm{Ji}$ ménez-Montero et al., 2012). Despite the fact that it is estimated that approximately 7,3 , or 2 cows need to be added to the reference population to achieve the same gain as from adding 1 bull, for traits with heritabilities of $0.1,0.3$, and 0.5, respectively (de Roos, 2011), this option may be relatively cost efficient because for some traits the cow phenotypes have already been recorded. An alternative strategy could be to contract herds to measure phenotypes for many traits under controlled circumstances (König and Swalve, 2009).

In each of those scenarios, an important question is which cows should be genotyped. From a practical point of view, one might just include all animals from a limited number of herds, regardless of their ancestry or performance. In our study, we were restricted to using cows with available genotypes. Our data mainly comprises batches of animals within research herds that were part of experiments (Ireland, Sweden, and the Netherlands), selection or control lines (UK), and bull dams (the Netherlands). It should be noted that the cross-validation applied in our study yields accuracies for a scenario where validation animals may originate from the same generation as the reference population. Still, because animals from different countries were included and because validation groups always were defined within countries, which reduces the relationship between evaluated animals and the reference population, it is not expected that the obtained accuracies are inflated due to the applied cross-validation approach. In fact, predicted accuracies obtained with the Daetwyler formula (Daetwyler et al., 2008; Daetwyler, 2009) were 0.35 and 0.34 for fat and protein, respectively, using heritabilities calculated in our study, assuming that the number of effective chromosome segments is 1,000 and that the reference population contains $90 \%$ of 1,609 cows. The predicted accuracy was close to our empirical estimate for fat yield and substantially greater than the empirical value for protein. Because the Daetwyler formula assumes that the evaluated individuals and the reference population are unrelated, this indicates that our accuracies were rather underestimated than overestimated.

From the perspective of DGV accuracy, the design of the reference population could be optimized such that it has a strong relationship with animals whose DGV are to be predicted, while at the same time the relationships among animals in the reference population design should be minimized (Pszczola et al., 2012a). With regard to the performance of the cows, it has been shown that sampling extreme cows (i.e., from both tails of the distribution of phenotypes) yields higher accuracy than selecting cows at random or only selecting the best cows (Jiménez-Montero et al., 2012). Selecting the best cows, in fact, yielded the lowest accuracy. This observation, together with the phenomenon of preferential treatment (Rensing et al., 2009), indicates that adding only elite female animals to the reference population (e.g., bull dams) may lead to very limited increase in accuracy and may, in fact, lead to biased estimates.

\section{CONCLUSIONS}

The developed bivariate BSSVS model was able to analyze 2 traits, even though all animals had measurement on only 1 of the 2 traits. The BSSVS model yielded consistently higher accuracy for fat yield compared with GREML, both for the univariate and bivariate analyses, whereas the accuracy of both models was similar for protein yield. The bivariate model revealed several additional QTL peaks compared with the single-trait 
models on either trait. In addition, the bivariate model showed a marginal increase in accuracy of genomic predictions for the cow traits $(0.01-0.05)$, whereas a higher increase is expected when adding more bulls to the analysis. Our results stress that the chosen value of priors in Bayesian genomic prediction models are especially important in small data sets.

\section{ACKNOWLEDGMENTS}

Three anonymous reviewers are thanked for their valuable comments on the manuscript. The authors acknowledge funding for the study from the Dutch Dairy Board (PZ; Zoetermeer, the Netherlands), AgentschapNL (The Hague, the Netherlands), and the RobustMilk project. The RobustMilk project is gratefully acknowledged for providing the data, in particular Donagh Berry (Teagasc Moorepark, Fermoy, Co. Cork, Ireland), Eileen Wall [Scottish Agricultural College (SAC), Edinburgh, UK; data collection funded by the Scottish Government], and Erling Strandberg [Swedish University of Agricultural Sciences (SLU), Uppsala, Sweden]. John Bastiaansen (Wageningen University, Wageningen, the Netherlands) is thanked for initial editing of the genotype data. The Irish Cattle Breeding Federation (Bandon, Co. Cork, Ireland) is gratefully acknowledged for providing bull DYD. The RobustMilk project is financially supported by the European Commission under the Seventh Research Framework Programme (Grant Agreement KBBE-211708). The content of this paper is the sole responsibility of the authors, and it does not necessarily represent the views of the Commission or its services.

\section{REFERENCES}

Ashwell, M. S., D. W. Heyen, T. S. Sonstegard, C. P. Van Tassell, Y. Da, P. M. VanRaden, M. Ron, J. I. Weller, and H. A. Lewin. 2004. Detection of quantitative trait loci affecting milk production, health, and reproductive traits in Holstein cattle. J. Dairy Sci. 87:468-475.

Banos, G., M. P. Coffey, R. F. Veerkamp, D. P. Berry, and E. Wall 2012. Merging and characterising phenotypic data on conventional and rare traits from dairy cattle experimental resources in three countries. Animal 6:1040-1048.

Berry, D. P., J. W. M. Bastiaansen, R. F. Veerkamp, S. Wijga, E. Wall, B. Berglund, and M. P. L. Calus. 2012. Genome-wide associations for fertility traits in Holstein-Friesian dairy cows using data from experimental research herds in four European countries. Animal 6:1206-1215.

Berry, D. P., L. Shalloo, A. R. Cromie, V. E. Olori, R. F. Veerkamp, P. Dillon, P. R. Amer, R. Evans, F. Kearney, and B. Wickham. 2007. The economic breeding index: A generation on. The Irish Cattle Breeding Federation. Accessed July 9, 2012. http://www.herdplus. ie/publications/files/economic_breeding_index.pdf.

Browning, S. R., and B. L. Browning. 2007. Rapid and accurate haplotype phasing and missing-data inference for whole-genome association studies by use of localized haplotype clustering. Am. J. Hum. Genet. 81:1084-1097.
Buch, L. H., M. K. Sørensen, P. Berg, L. D. Pedersen, and A. C. S $\varnothing-$ rensen. 2012. Genomic selection strategies in dairy cattle: Strong positive interaction between use of genotypic information and intensive use of young bulls on genetic gain. J. Anim. Breed. Genet. 129:138-151.

Calus, M. P. L. 2010. Genomic breeding value prediction: Methods and procedures. Animal 4:157-164.

Calus, M. P. L., Y. de Haas, M. Pszczola, and R. F. Veerkamp. 2013. Predicted accuracy of and response to genomic selection for new traits in dairy cattle. Animal 7:183-191.

Calus, M. P. L., H. A. Mulder, and J. W. M. Bastiaansen. 2011. Identification of Mendelian inconsistencies between SNP and pedigree information of sibs. Genet. Sel. Evol. 43:34.

Calus, M. P. L., and R. F. Veerkamp. 2011. Accuracy of multi-trait genomic selection using different methods. Genet. Sel. Evol. 43:26

Cromie, A. R., D. P. Berry, B. Wickham, J. F. Kearney, J. Pena, J. B. C. H. M. Van Kaam, N. Gengler, J. Szyda, U. Schnyder, M. P. Coffey, K. Moster, J. I. Weller, D. Abernethy, and R. Spelman. 2010. International Genomic Co-operation; Who, what, when, where, why and how? Pages 72-78 in Interbull Bulletin no. 42. Proc. of the Interbull International Workshop-Genomic Information in Genetic Evaluations, Riga, Latvia. Interbull, Uppsala, Sweden.

Daetwyler, H. D. 2009. Genome-wide evaluation of populations. PhD Thesis. Wageningen University, Wageningen, the Netherlands.

Daetwyler, H. D., B. Villanueva, and J. A. Woolliams. 2008. Accuracy of predicting the genetic risk of disease using a genome-wide approach. PLoS ONE 3:e3395.

de Haas, Y., M. P. L. Calus, R. F. Veerkamp, E. Wall, M. P. Coffey, H. D. Daetwyler, B. J. Hayes, and J. E. Pryce. 2012. Improved accuracy of genomic prediction for dry matter intake of dairy cattle from combined European and Australian data sets. J. Dairy Sci. 95:6103-6112.

de los Campos, G., J. M. Hickey, R. Pong-Wong, H. D. Daetwyler, and M. P. L. Calus. 2013. Whole-genome regression and prediction methods applied to plant and animal breeding. Genetics 193:327-345.

de Roos, A. P. W. 2011. Genomic selection in dairy cattle. PhD Thesis. Wageningen University, Wageningen, the Netherlands.

Ducrocq, V., and B. Besbes. 1993. Solution of multiple trait animal models with missing data on some traits. J. Anim. Breed. Genet. 110:81-92.

Fikse, W. F., and G. Banos. 2001. Weighting factors of sire daughter information in international genetic evaluations. J. Dairy Sci 84:1759-1767.

Garrick, D. J., J. F. Taylor, and R. L. Fernando. 2009. Deregressing estimated breeding values and weighting information for genomic regression analyses. Genet. Sel. Evol. 41:55.

Goddard, M. 2009. Genomic selection: Prediction of accuracy and maximisation of long term response. Genetica 136:245-257.

Harder, B., J. Bennewitz, N. Reinsch, G. Thaller, H. Thomsen, C. Kuhn, M. Schwerin, G. Erhardt, M. Forster, F. Reinhardt, and E. Kalm. 2006. Mapping of quantitative trait loci for lactation persistency traits in German Holstein dairy cattle. J. Anim. Breed. Genet. 123:89-96.

Hayes, B. J., P. J. Bowman, A. J. Chamberlain, and M. E. Goddard. 2009. Invited review: Genomic selection in dairy cattle: Progress and challenges. J. Dairy Sci. 92:433-443.

Jeffreys, H. 1961. The Theory of Probability. 3rd ed. Oxford University Press, Oxford, UK.

Jiménez-Montero, J. A., O. Gonzalez-Recio, and R. Alenda. 2012. Genotyping strategies for genomic selection in dairy cattle. Animal $6: 1216-1224$.

Kirkpatrick, B. W., X. Shi, G. E. Shook, and M. T. Collins. 2011 Whole-genome association analysis of susceptibility to paratuberculosis in Holstein cattle. Anim. Genet. 42:149-160.

Koenen, E. P. C., and R. F. Veerkamp. 1998. Genetic covariance functions for live weight, condition score, and dry-matter intake measured at different lactation stages of Holstein Friesian heifers. Livest. Prod. Sci. 57:67-77. 
König, S., and H. H. Swalve. 2009. Application of selection index calculations to determine selection strategies in genomic breeding programs. J. Dairy Sci. 92:5292-5303.

Legarra, A., and I. Misztal. 2008. Computing strategies in genomewide selection. J. Dairy Sci. 91:360-366.

Lund, M. S., A. P. W. de Roos, A. G. de Vries, T. Druet, V. Ducrocq, S. Fritz, F. Guillaume, B. Guldbrandtsen, Z. Liu, R. Reents, C. Schrooten, F. Seefried, and G. Su. 2011. A common reference population from four European Holstein populations increases reliability of genomic predictions. Genet. Sel. Evol. 43:43.

Mark, T., P. Madsen, J. Jensen, and W. F. Fikse. 2005. Short communication: Difficulties in estimating across-country genetic correlations for weakly linked bull populations. J. Dairy Sci. 88:33033305 .

Meuwissen, T. H. E., and M. E. Goddard. 2004. Mapping multiple QTL using linkage disequilibrium and linkage analysis information and multitrait data. Genet. Sel. Evol. 36:261-279.

Meuwissen, T. H. E., B. J. Hayes, and M. E. Goddard. 2001. Prediction of total genetic value using genome-wide dense marker maps. Genetics 157:1819-1829.

Ogorevc, J., T. Kunej, A. Razpet, and P. Dovc. 2009. Database of cattle candidate genes and genetic markers for milk production and mastitis. Anim. Genet. 40:832-851.

Pszczola, M., T. Strabel, H. A. Mulder, and M. P. L. Calus. 2012a. Reliability of direct genomic values for animals with different relationships within and to the reference population. J. Dairy Sci. 95:389-400.

Pszczola, M., T. Strabel, J. A. M. van Arendonk, and M. P. L. Calus. 2012b. The impact of genotyping different groups of animals on accuracy when moving from traditional to genomic selection. J Dairy Sci. 95:5412-5421.

Rensing, S., E. Pasman, and F. Reinhardt. 2009. Best use of conventional EBV of bull dams and combination with direct genomic values. Pages 123-126 in Interbull Bulletin no. 40. Proc. of the Interbull International Workshop-Genomic Information in Genetic Evaluations, Barcelona, Spain. Interbull, Uppsala, Sweden

Rodriguez-Zas, S. L., B. R. Southey, D. W. Heyen, and H. A. Lewin. 2002. Interval and composite interval mapping of somatic cell score, yield, and components of milk in dairy cattle. J. Dairy Sci. 85:3081-3091.

Ron, M., D. Kliger, E. Feldmesser, E. Seroussi, E. Ezra, and J. I. Weller. 2001. Multiple quantitative trait locus analysis of bovine chromosome 6 in the Israeli Holstein population by a daughter design. Genetics 159:727-735.
Schaeffer, L. R., J. W. Wilton, and R. Thompson. 1978. Simultaneous estimation of variance and covariance components from multitrait mixed model equations. Biometrics 34:199-208.

Sorensen, D., and D. Gianola. 2002. Likelihood, Bayesian and MCMC Methods in Quantitative Genetics. Springer Verlag, New York, NY

Tsuruta, S., I. Misztal, I. Aguilar, and T. J. Lawlor. 2011. Multipletrait genomic evaluation of linear type traits using genomic and phenotypic data in US Holsteins. J. Dairy Sci. 94:4198-4204.

van Binsbergen, R., R. F. Veerkamp, and M. P. L. Calus. 2012. Makeup of the genetic correlation between milk production traits using genome-wide single nucleotide polymorphism information. J. Dairy Sci. 95:2132-2143.

VanRaden, P. M., and P. G. Sullivan. 2010. International genomic evaluation methods for dairy cattle. Genet. Sel. Evol. 42:7.

VanRaden, P. M. 2008. Efficient methods to compute genomic predictions. J. Dairy Sci. 91:4414-4423.

Veerkamp, R. F., M. P. Coffey, D. P. Berry, Y. de Haas, E. Strandberg, H. Bovenhuis, M. P. L. Calus, and E. Wall. 2012. Genome-wide associations for feed utilisation complex in primiparous HolsteinFriesian dairy cows from experimental research herds in four European countries. Animal 6:1738-1749.

Verbyla, K. L., M. P. L. Calus, H. A. Mulder, Y. de Haas, and R. F. Veerkamp. 2010. Predicting energy balance for dairy cows using high-density single nucleotide polymorphism information. J. Dairy Sci. 93:2757-2764.

Verbyla, K. L., B. J. Hayes, P. J. Bowman, and M. E. Goddard. 2009. Accuracy of genomic selection using stochastic search variable selection in Australian Holstein Friesian dairy cattle. Genet. Res. (Camb.) 91:307-311

Wiener, P., I. Maclean, J. Williams, and J. Woolliams. 2000. Testing for the presence of previously identified QTL for milk production traits in new populations. Anim. Genet. 31:385-395.

Wiggans, G. R., T. S. Sonstegard, P. M. VanRaden, L. K. Matukumalli, R. D. Schnabel, J. F. Taylor, J. P. Chesnais, F. S. Schenkel, and C. P. Van Tassel. 2008. Genomic evaluations in the United States and Canada: A collaboration. Pages 347-353 in Proc. 36th ICAR Biennial Session held in Niagara Falls. International Committee for Animal Recording (ICAR), Rome, Italy.

Yang, J., B. Benyamin, B. P. McEvoy, S. Gordon, A. K. Henders, D. R. Nyholt, P. A. Madden, A. C. Heath, N. G. Martin, G. W. Montgomery, M. E. Goddard, and P. M. Visscher. 2010. Common SNPs explain a large proportion of the heritability for human height. Nat. Genet. 42:565-569. 